\title{
Characterisation of Biological Tissue by Ultrasound
}

\section{F. Hottier and M. Fink, Limeil-Brévannes}

(Laboratoires d'Electronique et de Physique Appliquée (LEP), Philips

Associate Member of EPS)

\begin{abstract}
The interaction of ultrasound with biological tissue is complex and involves scattering, absorption and refraction. The research discussed aims at providing clinically useful information from an analysis of reflected signals from the human body.
\end{abstract}

Ultrasonic waves are partially reflected and scattered by biological tissues. In clinical ultrasonic systems, an ultrasound transducer transmits a very short ultrasound pulse to the medium under examination and this same transducer is switched in reception mode and "listens" for the echoes caused by reflectors such as organ boundaries or small scatterers embedded in the tissue. The ultrasound velocity can be considered to be constant inside the body so that there exists a direct relationship between the echo time and the range of the scattered object, its direction being given by the direction in which the transducer is aimed. In the "A-mode" of presentation, the amplitude of the echoes are displayed as a function of their depth (i.e. of the time). In the B-mode, the echo amplitude modulates the electron beam of the display and the echoes are displayed as bright dots along a linear trace.

By moving the transducer along the body wall, and sending successive pulses through the body from different directions, an image of a tissue slice can be obtained. Thanks to this, the physician can see the contours of the organs, and different textural appearance of the image may give additional information about its state. The signal processing used in these imaging systems consists of taking the signal envelope and coding the echoes with grey levels according to their amplitude. In practice, the original echographic signal contains other information such as its frequency or its spec-

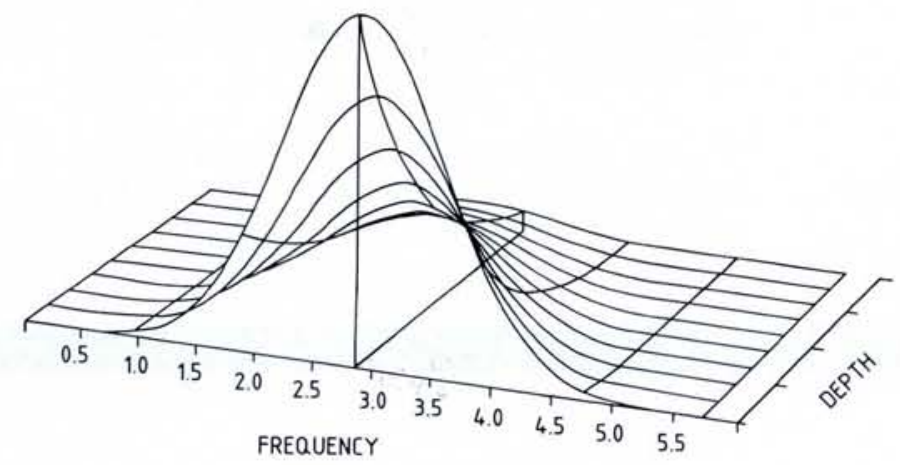

Fig. 1 - Spectral composition change due to a linearly frequency dependent attenuation.

tral content which can give access to a quantitative estimation of tissue acoustical parameters, for example its scattering properties or its attenuation. The knowledge of these parameters makes it possible to differentiate between pathologic and healthy organs in a clinical background.

The attenuation of ultrasound inside tissue is known to be a quality sensitive to the tissue pathology ${ }^{1}$ ). Short time Fourier analysis of the signal, gives a local estimation of the attenuation ${ }^{2,3}$ ) by estimating either the variations of the spectral content or the signal intensity measured in a narrow frequency band. Both estimations must be corrected for systematic errors arising from diffraction phenomena which are especially marked when using focussed transducers $^{4}$ ).

\section{Tissue Modelling}

A biological tissue is conveniently described by a discrete set of scatterers embedded in an attenuating medium. A short ultrasound pulse transmitted by the transducer is modified by two effects on its path to the scatterer which we define as being located at position $\vec{r}_{i}$ :

- a rather complicated diffraction phenomenon: the wideband pulse induces a diffraction pattern where the higher frequency components of the beam are more concentrated on the axis than the lower. It can be considered as a diffraction filter which is depth dependent; 


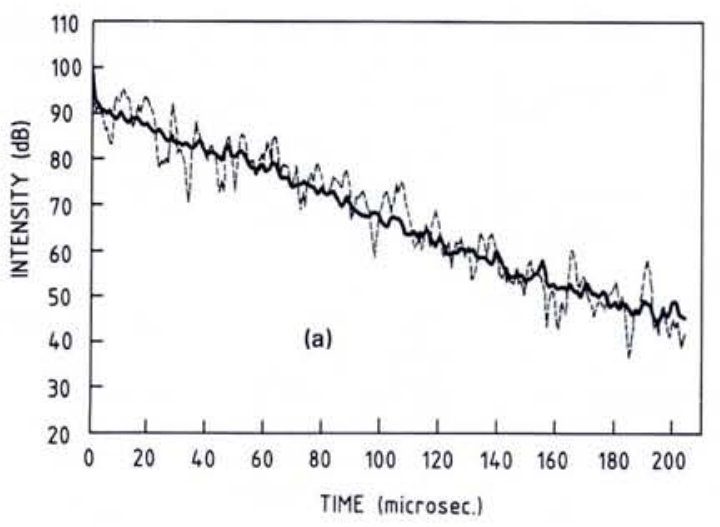

Fig. 3 -

a) Variation of the cen troid frequency versus the distance.

b) Variation of the signal intensity in three different narrow frequency bands (the transducer used to record the echographic signals is unfocussed).

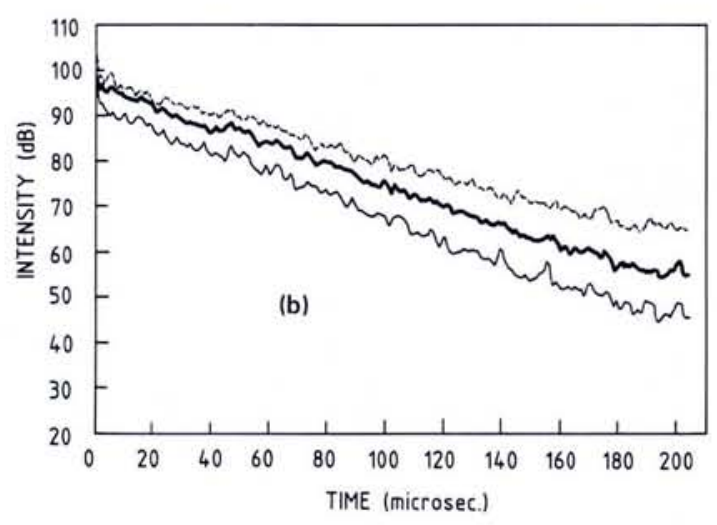

It is instructive to plot $S(f, d)$ as a function of time (Fig. 1). Not only is a general decrease of the signal intensity observed, but also a change in the spectral composition with enhancement of the low frequency components; this effect obviously stems from the lowpass attenuation filter.

To get a similar local spectral estimation of the echographic signal, a short time Fourier analysis has been made (Fig. 2): the signal is sampled by a sliding window and the corresponding spectrum modulus is computed for each window position. The snapshot of the successive spectra shows the same characteristics as Fig. 1.

\section{Attenuation Estimators}

Two approaches can be followed to get the attenuation from this representation. It can be shown that if the transducer spectrum has a gaussian envelope and the attenuation is linearly dependent on frequency (which is a rather good approximation for many biological tissues), the spectrum centroid frequen-

\section{UNIVERSITY OF BRISTOL DEPARTMENT OF PHYSICS}

Applications are invited for a two-year SERC funded research assistantship to work on the theory of the electronic and magnetic structure in $\mathrm{Ni}_{c} \mathrm{Fe}_{1-c}$ alloys with a focus on the INVAR region $(c \sim 0.36)$.

The salary scale is the usual SERC RA 1 A scale.

It is hoped that the successful applicant will be able to take up the position soon after 1 October 1984.

Applications should to be sent to:

Dr. B.L. Gyorffy,

HH Wills Physics Laboratory, Royal Fort, Tyndall Avenue, Bristol BS8 1TL, England. cy $f_{c}$ versus the propagation distance $d$ is a straight line whose slope is proportional to the $\beta$ parameter:

$$
f_{c}=-2 \beta c_{m} \sigma^{2} d
$$

$\sigma$ is the transducer spectrum variance and $c_{m}$ the medium ultrasound velocity.

In order to test this deduction, a set of 32 independent echographic A-lines coming from a test-object simulating the acoustic properties of a liver tissue has been used. Figure 3 a shows the centroid frequency variation versus the distance deduced from one A-line (dashed line) and from the average of the 32 A-lines (solid line). The noise observed on the first curve due to the random distribution of scatterers has been eliminated.

The attenuation law is also simply obtained by plotting the signal intensity in a narrow frequency band centred around $f_{\mathrm{B}}$ versus the distance. A quick look at expression (3) reveals that the quantity $\beta f_{\mathrm{B}}$ is simply proportional to the plot slope, the signal intensity being expressed in decibels. This parameter is plotted in Fig. 3b for three successive narrow frequency bands. The slope of these dif- ferent curves allows us to obtain an estimation of the two parameters $(\beta, n)$ of the attenuation law.

\section{Diffraction Correction in Attenuation Measurement}

The bias introduced by the depth dependent diffraction filtering effect can be simply explained by examining the diffraction impulse response, in the time domain, on the axis of a focussed transducer. Assume that a velocity impulse is applied to the transducer surface: as illustrated in Fig. 4, a scatterer located at the transducer focus is at the same distance from all the point sources of the radiating aperture, so that waves radiated from the transducer surface arrive with the same delay. The impulse response is brief and tends to a Dirac function; there is no filtering effect. In contrast, the propagation distances are significantly different in the transducer near and far fields which makes the duration of the impulse response longer and, as a result, the low frequency components of the transfer function are
KERNFYSISCH VERSNELLER INSTITUUT GRONINGEN, THE NETHERLANDS

\section{Post-doctoral Position in Theoretical Nuclear Physics}

The K.V.I. has a position at a post-doctoral level available for a young nuclear theorist. The initial appointment will be for a period of two years. Promotion to a permanent position thereafter is not excluded. The appointment will be with F.O.M., the Netherlands Foundation for Fundamental Research on Matter.

The Kernfysisch Versneller Instituut is a national research institute in nuclear physics, jointly sponsored by the University of Groningen and by F.O.M. It has a broad experimental and theoretical research programme on nuclear structure and heavy-ion physics.

Applicants for the above-mentioned position are requested to submit, as early as possible but not later than 1 November, 1984, a curriculum vitae, list of publications and the names of three persons who can provide letters of recommendations to:

Prof. A. van de Woude,

Kernfysisch Versneller Instituut, Zernikelaan 25,

9747 AA Groningen, the Netherlands. 

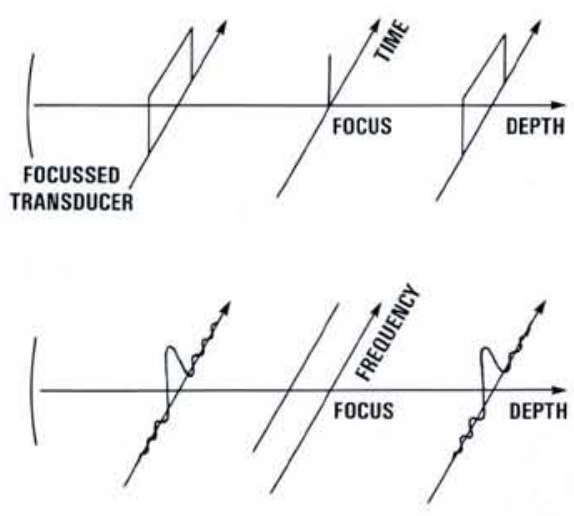

increased significantly. By taking into account the off-axis scatterers, a smoothing of the diffraction transfer function is obtained.

In order to correct the experimental spectra $S(f, d)$, a calibration procedure has been studied. A scattering phantom, immersed in water is illuminated by the transducer under study. The backscattered signal coming from a slice located slightly below the phantom surface is selected by using a temporal window, centred at the time $\tau=c_{\mathrm{w}} d$ where $c_{w}$ is the water ultrasound velocity; and the windowed signal power spectrum is computed. Then for the volume selected by the window, we obtain:

$$
S_{\text {cal }}(f, d)=K^{2}(f) .\left(U^{\prime}\right)^{2}(f) H^{4}(f, d)
$$

where $U^{\prime}(f)$ is the scattering response of the phantom. The effect of water attenuation is neglected. A mean diffraction filter is obtained by moving the transducer in a plane parallel to the selected slice. The same procedure is applied for different axial distances between the transducer and the phantom. It then gives the depth (time) varying dependence of the mean diffraction filter. In order to avoid attenuation variations within the phantom, the distance settings are adjusted in such a way that the sampled signal always comes from the same slice of the phantom, regardless of the selected axial distance. Figure 5 represents the 3-D plot of the mean time varying filter for a focussed transducer (diameter $=19 \mathrm{~mm}$, focus $=90 \mathrm{~mm}$ ), between $20 \mathrm{~mm}$ and $160 \mathrm{~mm}$. Time va-

Fig. 5 - Experimental 3-D plot of the diffraction mean time varying filter for a focus. sed transducer.

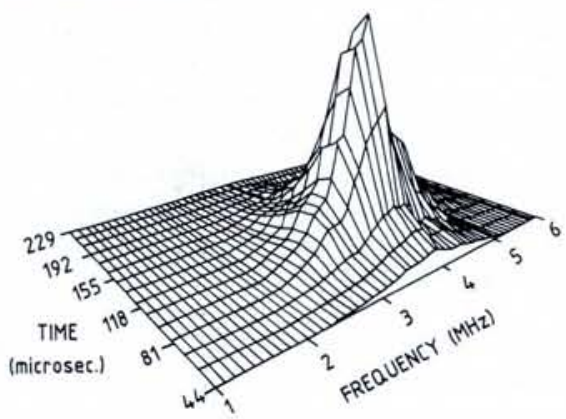

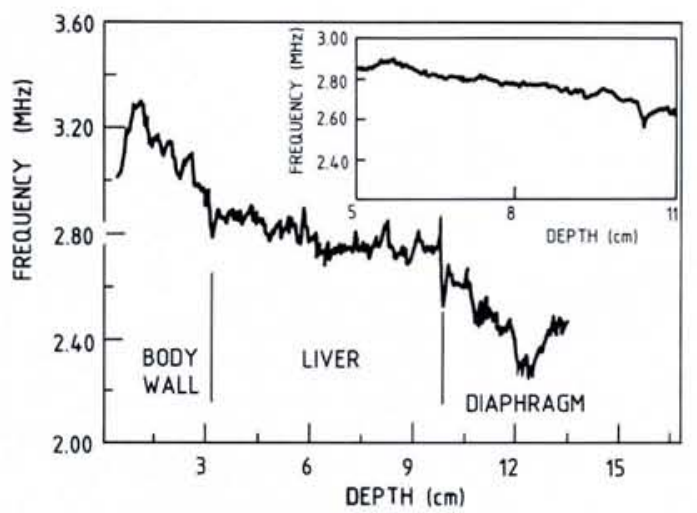

Fig. 4 (Far left) - Evolution of the diffraction impulse response on the axis of a focussed transducer.

Fig. 6 (Left) - Centroid frequency variation observed in the case of in vivo liver.

Fig. 7 (Right) - Images of a liver tissue slice:

Above: reflectivity image.

Centre: centroid mapping.

Below: narrow band signal intensity. rying effects of the diffraction filter are clearly shown on this plot: the signal intensity is increased around the focus, with a higher frequency content in the focal zone. Using these calibrated spectra the diffraction effect can be removed from the estimated running spectrum $S(f, d)$ by computing the corrected spectrum $C(f, d)$ :

$C(f, d)=\frac{S(f, d)}{S_{\mathrm{cal}}(f, d)}=\frac{U(f)}{U^{\prime}(f)} \exp \left(-4 \beta f^{n} d\right)$

The only remaining depth dependent filter is then due to the attenuation. Table 1 shows the efficiency of this correction: two sets of data have been used, one with a four centimeter thick phantom set slightly in front of the focal point, the second with the same phantom set behind this point.

\section{Experimental Results on in-vivo Data}

The digitized echographic A-line is selected by using an imaging $B$-scan unit linked to the acquisition system. Figure 6 shows the centroid frequency variation experienced by an A-line crossing the liver. Various attenuations associated with different biological tissues can be distinguished: the body wall presents a stronger attenuation than the liver. Inset is shown a curve resulting from an average of five such curves selected in a homogeneous part of the liver. The slope deduced from it gives an attenuation of $0.5 \mathrm{~dB} / \mathrm{cm} \mathrm{MHz}$.

The photographs correspond to a set of liver in-vivo data. The first one is a conventional reflectivity image (tissue area of about $3 \times 3 \mathrm{~cm}^{2}$ ) obtained through a software processing of 512

Table 1 - Correction efficiency recorded A-lines. A vein is easily seen in the bottom centre. A mapping of the centroid estimator resulting from the same set of data is shown on the next photograph. A profile of the centroid frequency along the upper white line is superimposed on the photographs and shows the decrease of this estimator with depth. The last photograph shows the mapping of the narrow band signal intensity, always with the same set of data. A comparison of these two last photographs illustrates the key features of these two attenuation estimators. The brightness variation, which accounts for the variance of the attenuation estimation is similar. It is associated with the distribution of scatterers inside the tissue which causes:

i) an amplitude variation of the echographic signal, adding then noise to the narrow band signal intensity estimator,

ii) variation of the spectrum frequency content, due mainly to interference phenomena, responsible for the noise of the centroid estimator.

In the case of a specular reflector, such as the vein present in the image, the behaviour of these two estimaturs looks different, the centroid being less affected by this inhomogeneity.

\section{Conclusion}

The main difficulty in tissue characterization is to develop analytical methods that will isolate the different interactions. At LEP, we have chosen to concentrate our effort on the estimation of tissue attenuation in order to provide clinically useful information, for example in the diagnosis of diffuse liver disease ${ }^{1}$ ).

(Cont. page 16)

\begin{tabular}{|c|c|c|c|c|}
\hline & \multicolumn{4}{|c|}{ Attenuation $(\mathrm{dB} / \mathrm{cm} \mathrm{MHz})$ deduced from } \\
\hline & \multicolumn{2}{|c|}{ Centroid frequency } & \multicolumn{2}{|c|}{ Signal intensity in narrow band } \\
\hline & $U$ & C & $U$ & C \\
\hline first data set & 0.46 & 0.63 & 0.68 & 0.66 \\
\hline second data set & 1.22 & 0.67 & 0.90 & 0.63 \\
\hline
\end{tabular}

$U:$ no diffraction correction.

C: diffraction correction. 
Methods based on a time frequency representation of the echographic signal have been considered and a particular effort has been made to understand and to correct the bias on the attenuation estimation due to the diffraction. It remains to be seen whether all these algorithms on clinical data enable quantitative measurements to be made thus providing new tools in medical diagnosis.

\section{REFERENCES}

1. Kuc R., IEEE Trans. Biomed. Eng. BME 27 (1980), 312-319.

2. Fink M., Hottier F. and Cardoso J.F., Ultrasonic Imaging 3 (1983), 117-135.

3. Hottier F., Fink M., Donjon J., Bernatets J.L. and Cardoso J.F., Proceedings of the 1st International Symposium on Medical Imaging and Image Interpretation, ISMIII 82, 269-274.

4. Cardoso J.F. and Fink M., Proceedings of the IEEE Ultrasonics Symposium (Atlanta), (1983), pp. 841-846.

\section{EUROPEAN SPACE AGENCY}

The European Space Agency seeks for its European Space Research and Technology Centre, Noordwijk

\section{One Staff position and possibly two fellowship positions in the domain of} PLANETARY SCIENCE

The Staff position will require that part of the time is devoted to project work related to ongoing space missions and study work for future missions. However, initially the main aim will be to initiate and carry out research.

Fellows can devote their time fully to research.

The research will be in line with planned ESA missions in planetary science, and the following research topics, with emphasis on experimental techniques, will be considered:

- remote sensing of atmospheres, including dust and ice constituents

- in-situ techniques for observations of grains, atoms/molecules and plasma, in order to study interaction processes

- collection and analysis of dust and ultimately, as a long terms aim, collection of material from primitive planetary bodies.

Applications should be directed to the Head of Personnel, ESTEC, Postbus 299 2200 AG Noordwijk, the Netherlands, including detailed curriculum vitae. For enquiries phone 1719-83308.

\section{NEW COLLABORATING SOCIETIES}

Under the terms of the decision taken by Council in March of this year, extending to European astronomical societies the possibility of becoming Collaborating Societies of the EPS, the following societies have applied and been accepted:

Società Astronomica Italiana Nederlandse Astronomenclub benefits of individual membership. cretariat in Geneva.
Members of these societies may now join the European Physical Society for an annual fee of Sw.Fr. 50.-. For this they [like other 4d) members, such as those who are members of the American Physical Society] are entitled to the full

Application should be made to the $\mathrm{Se}$ -

\section{NUCLEAR PHYSICS BOARD}

As a result of the recent elections, the full members of the Board of the Nuclear Physics Division over the next three years will be as shown opposite:

The names of the Chairman and Secretary will be announced later.

Note: The October issue of Europhysics News is a Meetings issue
R. Bergere, CEA, Saclay

P. Glaudermans, University, Utrecht

G. Goldring, Weizmann Institute, Tel Aviv

I. Iori, Institute of Physics, Milan

P. Lieb, University, Göttingen

G. Morrison, University, Birmingham

I. Sick, University, Basel

P. von Brentano, University, Cologne

H. Zingl, University, Graz
G. Tibell, Gustaf Werners Inst., Uppsala

\section{PLASMA PHYSICS}

The result of the recent ballot in the Plasma Physics Division is given below: Full Members of the Board:

K. Appert, CRPP, Lausanne

R. Behrisch, IPP, Garching

W. Bœtticher, University, Hannover

F. Engelmann, NET, Garching

A. Gibson, JET, Abingdon

J. Jacquinot, JET, A bingdon

V. Jensen, Risø Nat. Lab., Roskilde

M. Kaufmann, IPP, Garching

$H$. de Kluiver, FOM, Nieuwegein

R.V.Sagdeev, Inst. of Space Research, Moscow

D.C. Schram, Univ. of Techn.

Eindhoven

D. Sweetman, Culham Lab., Abingdon

The Division also approved the proposed change in the Statutes whereby the outgoing Chairman may serve a further three-year term even though he may already have served on the Board for two consecutive terms.
EPS Divisions, Sections and Group Astronomy and Astrophysics Division

Solar Physics Section
Atomic and Molecular Physics Division Atomic Spectroscopy Section Chemical Physics Electronic and Atomic Collisions Molecular Physics Computational Physics Group

Condensed Matter Division Liquids Section

Low Temperature Physics Section Macromolecular Physics Magnetism Metal Physics Semiconductors and insulators Surfaces and Interfaces High Energy \& Particle Physics Division Nuclear Physics Division Optics Division Optics Division
Plasma Physics Division Quantum Electronics Division
Europhysics News is the official journal of the European Physical Society which comprises 29 National Socie ties, Academies and Group, over 3500 Individual Members and 66 Associate Members. Governing bodies of EPS are the General Meeting, Council and an elected Executive Committee responsible for detailed policy. EPS promotes the collaboration of physicists throughout Europe, organising and harmonising conferences and publications, improving physics education, encou raging physics applications, awarding scholarships to sponsored schools in Erice. EPS publishes in addition to EN, Europhysics Conference Abstracts, E. Ed. News and, in collaboration with The institute of Physics (UK) the European Journal of Physics. Individual Members receive EN free of charge (price to institutions: Sw.Fr. receive EN free of charge (price to institutions: Sw.Fr.
90 . - a), rebates on the price of many publications and on conference fees. Annual EPS membership fee for Individual Members belonging to an EPS member society is: Sw.Fr, 40.-; independent members: Sw.Fr. 120.; members of a Collaborating Society, e.g. the American Physical Society: Sw.Fr. 50. (\$25).

\section{Editor: E.N. Shaw}

Meetings Compilation: W.S. Newman

Editorial Board:

K. Appert, A. Baratoff, B. Jacrot,

G.R. Macleod, A. Maeder, J. Muller

Editorial and Advertising Office at the EPS Secretariat

Address: EUROPEAN PHYSICAL SOCIETY P. O. Box 69 , CH-1213 Petit-Lancy 2 Switzerland

Telephone: Geneva (22) 931130

Telex : $\mathbf{4 2 3} \mathbf{4 5 5}$ dema ch

Cables: europhys genève

Printed by: Pfirter frères sa $\mathrm{CH}-1213$ Petit-Lancy/Switzerland 